\title{
Antibiotic Resistance Pattern of Hospital Isolates of Staphylococcus aureus in Mashhad-Iran During 2009 - 2011
}

\author{
Ahmadreza Zarifian ${ }^{1}$, Ali Sadeghian ${ }^{1}$, Hamid Sadeghian ${ }^{1,2}$, Kiarash Ghazvini ${ }^{1}$, Hadi \\ Safdari ${ }^{1,2, *}$ \\ ${ }^{1}$ Antimicrobial Resistance Research Center, Mashhad University of Medical Sciences, Mashhad, IR Iran \\ 2 Department of Laboratory Sciences, School of Paramedical Sciences, Mashhad University of Medical Sciences, Mashhad, IR Iran \\ *Corresponding author: Hadi Safdari, Antimicrobial Resistance Research Center, Mashhad University of Medical Sciences, Mashhad, IR Iran, Tel.: +98-5117112611, Fax: +98-5117112596, \\ E-mail:safdarih@mums.ac.ir.
}

Received: April 16, 2012; Revised: May 02, 2012; Accepted: May 13, 2012

\begin{abstract}
Background: Staphylococcus aureus is a major human pathogen. Due to high prevalence of S. aureus infections and increasing resistance to antibiotics, physicians have been facing problems in choosing the appropriate empirical antibiotic therapy for such infections.

Objectives: The aim of this study was to determine the pattern of antibiotic resistance in S. aureus clinical isolates of Mashhad Quaem Hospital between 2009 and 2011.

Materials and Methods: In this study 170 isolates of Staphylococcus aureus were identified in laboratory. The specimens(including 82 urine, 43 wound, 37blood, 8 stool samples) were collected and examined by standard diagnostic methods. Determination of S. aureus sensitivity to antibiotics was performed using standard disc diffusion method. The antibiotic sensitivity of bacteria were reported according to the clinical laboratory standards institute (CLSI) manual as sensitive (S), intermediate (I) and resistant (R).

Results: Among tested antibiotics, Staphylococci isolates were highly resistant against Ceftazidime (94\%), followed by Penicillin (91\%), Ampicillin (82\%), Cefotaxime (65\%), Erythromycin (60\%), and Oxacillin (43\%). Nearly all strains were susceptible to tested Vancomycin. Conclusions:Our results are similar tothe reports from other parts of Iran. According to this study, resistancepattern amongStaphylococcus aureus strains were widespread in Quaem hospital. The implication of this high resistance is that Staphylococcus aureus infections should be treated more consciously and not with Penicillin and other ineffective antibiotics.
\end{abstract}

Keywords: Antibiotics, Antineoplastic Agents; Drug Resistance; Staphylococcus aureus

\section{Background}

Staphylococcus aureus is a bacterium that colonizes both in the community and hospital settings (1). Globally, S. aureus is one of the principal microorganisms involved in nosocomial infections. Indubitably, the fact that makes this pathogen a cure-challenging germ is its innate capacities to endure hard conditions such as high salt concentrations, high temperatures, extremes $\mathrm{pH}$, survival in the presence of many antibiotics and disinfectants and even in macrophages (2). The prevalence of Staphylococcus aureus resistance to conventional antibiotics has been highly increasing in some hospitals. The use of wide spectrum of antibiotics in the hospital environment, leads to the development of increased resistance to these antimicrobial agents. This increasing prevalence of bacterial resistance to antibiotics, are mostly associated with the extensive use of antimicrobial agents, may result in insufficiency of the selected substances to combat some bacterial infections $(1,3)$.

The feature which makes $\mathrm{S}$. aureus a distinct pathogen is its competence in acquiring resistance against new drugs. This ability is mainly due to its plasmids, which carry an extensive range of drug-resistant genes (4). In Staphylococci, after inactivation of Penicillin by penicillinase, an enzyme that is coded by plasmid, methicillin will be inactivated. Resistanceto this semi-synthetic $\beta$-lactamase resistant Penicillin was reported in 1961 in London (5). Many studies have been conducted since then and suggested a steady increase in the prevalence of Methicillin Resistant Staphylococcus aureus (MRSA) from late 1970s until now. They suggested that MRSA is increasingly causing infection in hospitals and community with higher mortality and morbidity than infections due to MSSA (4-6). In 1997, decreased susceptibility to Vancomycin, the drug of last resort against MRSA 
infections, was reported followed by full resistance in $2002(3,7,8)$. In the word of increasing resistance of Staphylococcus aureus to antibiotics, especially to routine ones, physicians have faced problems in treating Staphylococcus aureus infections.

\section{Objectives}

The purpose of the study was to determine the antibiotic susceptibility pattern in Staphylococcus aureus isolates in Quaem hospital of Mashhad, from 2009 to 2011.

\section{Materials and Methods}

From September 2009 to March 2011, a total number of 2527 samples were collected from patients attending to Quaem hospital, Mashhad. Among these samples (urine, blood, wound and stool) 170 were identified as Staphylococcus aureus infected samples. For bacterial isolation and identification, the specimens were cultured according to standard methods. Culture media were incubated at $37^{\circ} \mathrm{C}$ for 18 hours under sterile circumstances. The isolates were identified using motility test, colony morphology, Gram staining, and biochemical tests such as catalase test, coagulase test, sensitivity to novobiocin, mannitol fermentation, and DNase tests. In order to determine the antimicrobial resistance pattern of isolates, we used 0.5 McFarland suspensions of bacteria on Mueller-Hinton's media. Disc diffusion susceptibility test was performed using seven antibiotic discs (i.e. Vancomycin, Ampicillin, Cefotaxime, Erythromycin, Penicillin, Oxacillin and Ceftazidime). The results reported after 18 hours incubation at $37^{\circ} \mathrm{C}$ according to the Clinical Laboratory Standards Institute (CLSI) (9).

\section{Results}

In this study Staphylococcus aureus was ranked as the second cause of hospital infections. Out of 2527 clinical samples, 170 (6.7\%) were positive for Staphylococcus aureus. The total of 2527 specimens was obtained from different parts of the body. Most of isolated S. aureus was collected from wound (12.9\%) while the least was from urine (5.6\%) (Table 1).

\begin{tabular}{lll}
\hline \multicolumn{2}{l}{ Table 1. Occurrence of S. aureus from Different Clinical Samples } \\
\hline Source & Number & S. aureus Isolated Numbers, No. (\%) \\
\hline Urine & 1457 & $82(5.6 \%)$ \\
Blood & 661 & $37(5.6 \%)$ \\
Wound & 334 & $43(12.9 \%)$ \\
Stool & 75 & $8(10.7 \%)$ \\
Total & 2527 & $170(6.7 \%)$ \\
\hline
\end{tabular}

1457 urine, 661 blood, 334 wound and 75 stool specimens were cultured. Staphylococcus aureus were isolated from 82 urine specimens (5.6\%), 37 blood specimens (5.6\%), 43 wound specimens (12.9\%) and 8 stool specimens (10.7\%). Most of isolates detected as $S$. aureus were collected from urine $(\mathrm{n}=82)$ and the least number were isolated from stool samples $(n=8)$. The degree of sensitivity of $S$. aureus isolates to antibiotics, following the disc diffusion method was analyzed according to CLSI 2007 (9) (Table 2).

Table 2. Sensitivity of S. aureus Isolates to Different Antibiotics Using Disc Diffusion Method

\begin{tabular}{llllll}
\hline Oxacillin & Erythromycin & Cefotaxime & Ampicillin & Penicillin & Ceftazidime \\
\hline $\mathbf{4 6} \%$ & $43 \%$ & $39 \%$ & $38 \%$ & $7 \%$ & $5 \%$ \\
\hline
\end{tabular}

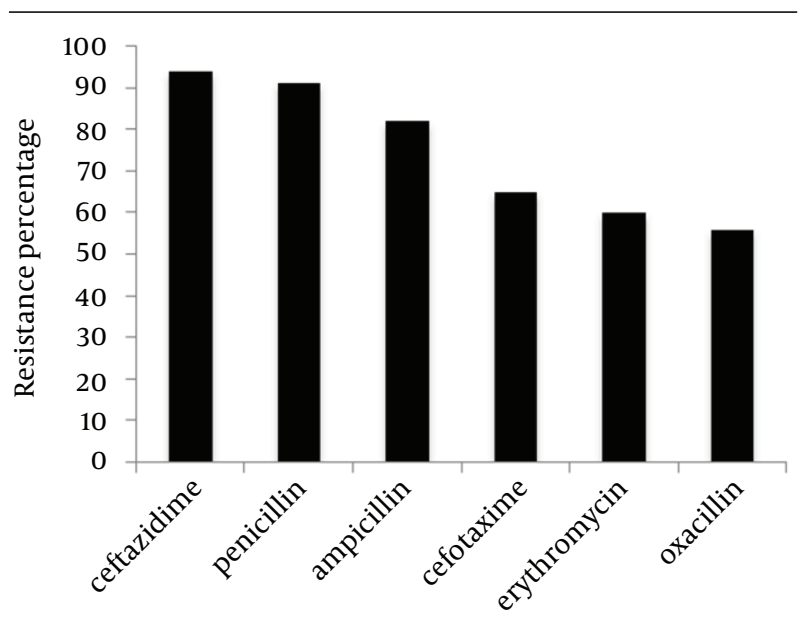

Figure 1. Resistance Percentage of S. aureus Isolates Against Various Antibiotics

\section{Discussion}

S. aureus is a major cause of hospital infections, which has been proved by many other studies $(10,11)$. Over past decades, the frequency of antimicrobial resistance and its association with serious infectious disease has been a global public health concern. Antimicrobial resistance resulted in increases in the number of illnesses, death and health-care costs (12). In this study, out of 2527 analyzed samples, 170 (6.7\%) were positive for Staphylococcus aureus with the most occurrences in wound (12.9\%), followed by stool specimens (10.7\%) and the least amount were reported in urine samples (5.6\%). This is in line with previous reports in which S. aureus was the most common organism isolated from wound samples (13-15). The results of this study indicated that S. aureus isolates showed the least resistance against Oxacillin (43\%) comparing other tested antibiotics. This suggests that Oxacillin can be an 
appropriate drug for S. aureus infections treatment. On the other hand, in another study done in Asia, high levels of Oxacillin resistance (82.1\%) were reported (16). Similarly, Pourakbari et al. reported 79\% resistance of S. aureus against Oxacillin in Tehran, Iran (17).

We expected higher Penicillin resistance rate in our isolates because other studies show more Penicillin resistance compared to our study $(14,18,19) .60 \%$ Erythromycin resistance was reported in this study, which is similar to another study conducted in Mashhad with 52.3\% resistance to Erythromycin (19). Furthermore, Pai et al. reported $45.9 \%$ Erythromycin resistance (18). This was in accordance with the Indian study in which Ahmed et al. recorded $74 \%$ resistance of MRSA isolates to Erythromycin (20). Although disk diffusion method is unable to generate the minimum inhibitory concentration (MIC) value (i.e. not quantitative as is the main disadvantage of this method), this method is a relatively easy and has been currently standardized in clinical settings by CLSI. In this study, we recorded $82 \%$ and $65 \%$ resistance of S. aureus isolates to Ampicillin and Cefotaxime, respectively. Nevertheless Naderinasab et al. reported 97.7\% Ampicillin resistance and 51.2\% Cefotaxime resistance in Imam Reza and Quaem hospitals in Mashhad (19). In conclusion, S. arouses is the major cause of nosocomial infections and the prevalence of antibiotic resistance among isolates is very high among patients referred to Quaem hospital, and this issue should be taken into consideration.

\section{Acknowledgements}

We are grateful to Mashhad University of Medical Sciences for their financial support.

\section{Authors' Contribution}

All authors contributed equally to this manuscript.

\section{Financial Disclosure}

The authors declare that there are no conflicts of interest.

\section{Funding/Support}

This study was supported by Mashhad University of Medical Sciences.

\section{References}

1. Holden MT, Feil EJ, Lindsay JA, Peacock SJ, Day NP, Enright MC et al. Complete genomes of two clinical Staphylococcus aureus strains: evidence for the rapid evolution of virulence and drug resistance. Proc Natl Acad Sci USA. 2004;101(26):9786-91.

2. Talaro KP, Talaro A. Foundations in Microbiology. 4 ed. New York,
US: McGraw Hill; 2002.

3. Chambers HF. The changing epidemiology of Staphylococcus aureus? Emerg Infect Dis. 2001;7(2):178-82.

4. Moellering RC Jr. MRSA: the first half century. J Antimicrob Chemother. 2012;67(1):4-11.

5. Kluytmans JAJW, Diederen BMW. Control of Healthcare-Associated Methicillin-ResistantStaphylococcus aureus. In: Gould IM, Meer JWM, editors. Antibiotic Policies: Fighting Resistance. Springer US; 2008. p. 253-69.

6. Rehm SJ.Staphylococcus aureus: the new adventures of a legendary pathogen. Cleve Clin J Med. 2008;75(3):177-80, 83-6, 90-2.

7. Blot SI, Vandewoude KH, Hoste EA, Colardyn FA. Outcome and attributable mortality in critically Ill patients with bacteremia involving methicillin-susceptible and methicillin-resistant Staphylococcus aureus. Arch Intern Med. 2002;162(19):2229-35.

8. Hiramatsu K, Hanaki H, Ino T, Yabuta K, Oguri T, Tenover FC. Methicillin-resistant Staphylococcus aureus clinical strain with reduced vancomycin susceptibility. J Antimicrob Chemother 1997;40(1):135-6.

9. Clinical and Laboratory Standards Institute. Performance standards for antimicrobial susceptibility testing. Seventeenth informational supplement. Wayne, PA: CLSI; 2007 [updated January 2007]; Available from: http://www.microbiolab-bg.com/CLSI.pdf.

10. Chang S, Sievert DM, Hageman JC, Boulton ML, Tenover FC, Downes FP, et al. Infection with vancomycin-resistant Staphylococcus aureus containing the vanA resistance gene. $N$ Engl J Med. 2003;348(14):1342-7.

11. Hiramatsu K, Aritaka N, Hanaki H, Kawasaki S, Hosoda Y, Hori S, et al. Dissemination in Japanese hospitals of strains of Staphylococcus aureus heterogeneously resistant to vancomycin. Lancet. 1997;350(9092):1670-3.

12. Cosgrove SE, Sakoulas G, Perencevich EN, Schwaber MJ, Karchmer AW, Carmeli Y. Comparison of mortality associated with methicillin-resistant and methicillin-susceptible Staphylococcus aureus bacteremia: a meta-analysis. Clin Infect Dis. 2003;36(1):53-9.

13. Tirabassi MV, Wadie G, Moriarty KP, Garb J, Konefal SH, Courtney RA, et al. Geographic information system localization of community-acquired MRSA soft tissue abscesses. J Pediatr Surg. 2005;40(6):962-5.

14. Pai V, Rao VI, Rao SP. Prevalence and Antimicrobial Susceptibility Pattern of Methicillin-resistant Staphylococcus Aureus [MRSA] Isolates at a Tertiary Care Hospital in Mangalore, South India. J Lab Physicians. 2010;2(2):82-4.

15. Yinusa W, Onche II, Thanni LO. Short-term antibiotic prophylaxis in implant surgery: a comparison of three antibiotics. Niger Postgrad Med J. 2007;14(2):90-3.

16. Chung DR, Song JH, Kim SH, Thamlikitkul V, Huang SG, Wang H et al. High prevalence of multidrug-resistant nonfermenters in hospital-acquired pneumonia in Asia. Am J Respir Crit Care Med. 2011;184(12):1409-17.

17. Pourakbari B, Sadr A, Ashtiani MT, Mamishi S, Dehghani M, Mahmoudi S, et al. Five-year evaluation of the antimicrobial susceptibility patterns of bacteria causing bloodstream infections in Iran.J Infect Dev Ctries. 2012;6(2):120-5.

18. Lowy FD. Antimicrobial resistance: the example of Staphylococcus aureus. J Clin Invest. 2003;111(9):1265-73.

19. Naderinasab M, TavakolAfshari J, Nazem M, FatehManesh P, Faramarzi H, Khoda Doost MA. Determination of Methicillin resistant Staphylococcus aureus via phenotypic methods and mecA gene study by multiplex PCR. Med J Mashhad U Med Sci. 2005;48:7-16.

20. Ahmed MO, Elramalli AK, Amri SG, Abuzweda AR, Abouzeed YM. Isolation and screening of methicillin-resistant Staphylococcus aureus from health care workers in Libyan hospitals. East Mediterr Health J. 2012;18(1):37-42. 